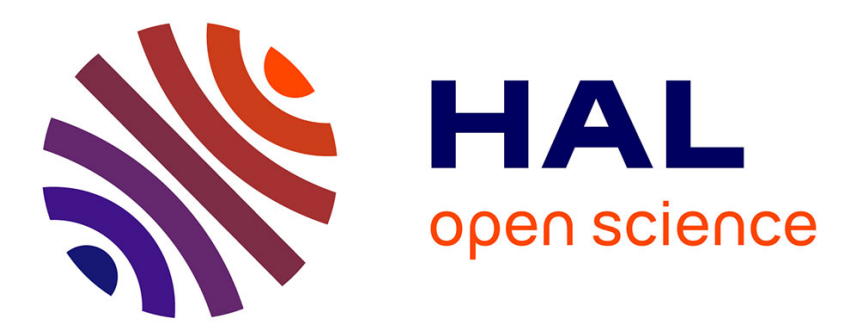

\title{
Sub-micron resolution selected area electron channeling patterns
}

\author{
Julien Guyon, H. Mansour, Nathalie Gey, M. A. Crimp, S. Chalal, Nabila \\ Maloufi
}

\section{> To cite this version:}

Julien Guyon, H. Mansour, Nathalie Gey, M. A. Crimp, S. Chalal, et al.. Sub-micron resolution selected area electron channeling patterns. Ultramicroscopy, 2015, 149, pp.34-44. 10.1016/j.ultramic.2014.11.004 . hal-01514962

\section{HAL Id: hal-01514962 \\ https://hal.univ-lorraine.fr/hal-01514962}

Submitted on 13 Dec 2019

HAL is a multi-disciplinary open access archive for the deposit and dissemination of scientific research documents, whether they are published or not. The documents may come from teaching and research institutions in France or abroad, or from public or private research centers.
L'archive ouverte pluridisciplinaire HAL, est destinée au dépôt et à la diffusion de documents scientifiques de niveau recherche, publiés ou non, émanant des établissements d'enseignement et de recherche français ou étrangers, des laboratoires publics ou privés. 


\title{
Sub-micron resolution selected area electron channeling patterns
}

\author{
J. Guyon ${ }^{\mathrm{a}, \mathrm{b}}$, H. Mansour ${ }^{\mathrm{a}}$, N. Gey ${ }^{\mathrm{a}, \mathrm{b}}$, M.A. Crimp ${ }^{\mathrm{c}}$, S. Chalal ${ }^{\mathrm{d}}$, N. Maloufi ${ }^{\mathrm{a}, \mathrm{b}, *}$ \\ a Laboratoire d'Étude des Microstructures et de Mécanique des Matériaux (LEM3), UMR CNRS 7239, Université de Lorraine, 57045 Metz, France \\ ${ }^{\mathrm{b}}$ Laboratory of Excellence on Design of Alloy Metals for low-mAss Structures (DAMAS), Université de Lorraine, France \\ ${ }^{\mathrm{c}}$ Department of Chemical Engineering \& Materials Science, Michigan State University, East Lansing, MI 48824, USA \\ ${ }^{\mathrm{d}}$ Carl Zeiss S.A.S, Division Microscopie et Systèmes, 100, route de Versailles, 78161 Marly le Roi, France
}

\section{A R T I C L E I N F O}

\section{Keywords:}

Rocking beam

SACP

EBSD

ECCI

FEG-SEM

Gemini column

\begin{abstract}
A B S T R A C T
Collection of selected area channeling patterns (SACPs) on a high resolution FEG-SEM is essential to carry out quantitative electron channeling contrast imaging (ECCI) studies, as it facilitates accurate determination of the crystal plane normal with respect to the incident beam direction and thus allows control the electron channeling conditions. Unfortunately commercial SACP modes developed in the past were limited in spatial resolution and are often no longer offered. In this contribution we present a novel approach for collecting high resolution SACPs (HR-SACPs) developed on a Gemini column. This HR-SACP technique combines the first demonstrated sub-micron spatial resolution with high angular accuracy of about $0.1^{\circ}$, at a convenient working distance of $10 \mathrm{~mm}$. This innovative approach integrates the use of aperture alignment coils to rock the beam with a digitally calibrated beam shift procedure to ensure the rocking beam is maintained on a point of interest. Moreover a new methodology to accurately measure SACP spatial resolution is proposed. While column considerations limit the rocking angle to $4^{\circ}$, this range is adequate to index the HR-SACP in conjunction with the pattern simulated from the approximate orientation deduced by EBSD. This new technique facilitates Accurate ECCI (A-ECCI) studies from very fine grained and/or highly strained materials. It offers also new insights for developing HR-SACP modes on new generation high-resolution electron columns.
\end{abstract}

\section{Introduction}

Since they were first identified by Coates [1] and subsequently interpreted by Booker et al. [2] using a block wave formulation, electron channeling patterns (ECPs) formed from backscattered electrons have been recognized as an effective tools for determining crystal orientations in a scanning electron microscope. In their general form, ECPs are collected at low magnification from single crystals or from very large grains of a polycrystalline material, where the scanning motion of the electron beam leads to a variation in the angle between the incoming electron beam and the crystal lattice planes as a function of position on the sample. These ECPs are then simply collected by imaging with the backscattered electrons. Depending on the microscope, the angular range of ECPs is between $3^{\circ}$ and $25^{\circ}$. However, the spatial resolution is quite poor $(\sim 1 \mathrm{~mm})$. Consequently, in this configuration, ECPs cannot be used to determine crystal orientations from

\footnotetext{
*Corresponding author at: Laboratoire d'Étude des Microstructures et de Mécanique des Matériaux (LEM3), UMR CNRS 7239, Université de Lorraine, 57045 Metz, France.

E-mail address: nabila.maloufi@univ-lorraine.fr (N. Maloufi).
}

most polycrystalline materials, which would typically have much finer grain sizes [3].

To overcome this spatial resolution limitation, the selected area electron channeling pattern (SACP) technique was developed, which reduces the area from which a pattern is collected by nominally rocking the electron beam at a point. Different procedures have been proposed to rock the beam in $\operatorname{TEM}^{1}[4-6]$ or to obtain SACPs in SEM [7-9]. The basic approach is to use two coils that are driven separately to rock the beam on an area of interest [10]. In the past, to produce SACPs several SEM microscope configurations have been developed, and due to the configuration, the area from which the pattern is collected can vary significantly in size. Practical issues encountered include: (1) the rocking point is not coincident with the surface of the sample and (2) spherical aberration in the final objective lens deflects the electron beam from the rocking point. Some microscopes have made corrections to improve the spatial resolution of the SACP (including the

\footnotetext{
${ }^{1}$ Rocking beam diffraction has been proposed in TEM by Vincent and Midgley [4] using the dark field scan coils in a circular hollow cone above the sample. This technique, termed "Double conical beam-rocking system", allows the measurement of integrated electron diffraction intensities with reduced dynamical effects and opens the way to crystal structure and symmetry determination [5,6].
} 
CamScan 44 and more recently the Tescan Mira instruments [11], leading to the ability to collect SACPs from relatively small areas, with claims in the range of $5-10 \mu \mathrm{m}$ for an angular range of $\sim 20^{\circ}$ ). However, the spatial resolutions have not been effectively demonstrated in the literature.

Since the early 1990s the ECP/SACP technique has been overshadowed by the development of electron backscattered diffraction (EBSD) pattern analysis. This is because EBSD offers a number of advantages over ECP, primarily good spatial resolution $(\sim 30 \mathrm{~nm})$ and very high data acquisition rates ( $>500$ orientations per second) [12-16]. Because of the advantages of EBSD, the use of the electron channeling phenomenon for determining crystal orientation has become uncommon. Despite this, there is one application where SACP offers significant advantages over EBSD, that being in relation to the imaging of dislocations and stacking faults in the near surface region of bulk samples using electron channeling contrast imaging (ECCI). In recent years, the use of ECCI has become more common, with a significant number of groups carrying out studies on a variety of materials [11,17-27]. The reason SACPs have an advantage over EBSDs for conducting ECCI studies is that in order to carry out quantitative studies, it is necessary to control the electron channeling conditions (in the same manner that specific diffracting "two-beam" conditions are used in diffraction contrast TEM analysis). The angular accuracy of EBSD is typically limited to about $1-2^{\circ}$ [28-31], and does not readily give the orientation with respect to the incident beam direction (optic axis). In contrast, SACPs directly display patterns with respect to the incident beam direction and does so with accuracies in the range of $0.1^{\circ}$, suitable for determining not only the specific set of planes used for imaging ( $g$-vector), but also for quantifying the deviation from the Bragg condition ( $s$ or $\omega$ ).

Unfortunately nearly all recently developed microscope configurations, particularly those with the field emission guns necessary for ECCI, preclude the ability to readily collect SACPs. As a consequence, many ECCI studies can only be considered semiquantitative as they do not consider the specific imaging conditions. As an example, high quality ECC images of dislocations using the Gemini column have been shown. However, because this column is not equipped with a dedicated SACP mode, the approximate channeling conditions have been estimated based on EBSD patterns [26]. Other ECCI studies, using FEI field emission gun columns, have been limited to single crystals [32-34].

In this paper, we present in detail a new approach for collecting SACPs on the Gemini column without further modifications. This approach results is the first report of sub-micron spatial resolution SACP, with demonstrated $500 \mathrm{~nm}$ resolution, which we term "High Resolution SACP". The resolution is demonstrated by a new, robust procedure for accurately determining the area that the SACP is collected from. This new approach to collecting SACP opens a new path towards improved SACP resolution and quantitative ECCI [35], not only with the Gemini electron column, but also with other new generation high-resolution electron columns.

\section{Methodology}

An innovative procedure has been developed to rock the electron beam on the high resolution Gemini electron column, which has no dedicated SACP mode. This procedure creates an SACP pivot point by combining control of the aperture alignment coils and the scanning coils, using them in a manner quite different from their primary functions. The beam is first deflected from and then brought back parallel to the optic axis by the aperture alignment coils; after the action of the scanning coils and the objective lens, the electron beam strikes the specimen surface at an angle. Because the off-axis beam is deflected in the scanning coils, the beam does not strike the sample at the optic axis. Thus, beam shift corrections controlled by the scanning coils are applied to ensure that the tilted beam strikes the sample at the intersection point of the sample surface and the optic axis; this point is the pivot point necessary for SACP. For a comprehensive understanding of this rocking beam procedure, it is important to review the specific configuration of the Gemini column and clarify the primary functions of the coils we used to rock the beam in this study.

\subsection{Gemini column in conventional scanning mode}

Simplified schemes of this column under different functionalities are given in Fig. 1. Unlike many scanning electron microscopes, the beam aperture in the Gemini column is not controlled by a mechanically selectable and aligned aperture, but instead by a series of coils that deflect the electron beam to the appropriate sized aperture hole in a multihole aperture (MA). That is, the beam is moved (in most cases off-axis) to the desired aperture, rather than the aperture being moved to and centered around the beam. This aperture control is accomplished by two sets of deflection coils. The first (upper) set deviates the beam to a selected hole (gun alignment coils, GC) and the second set (lower) brings the beam back to and parallel to the optic axis (aperture alignment coils, ApC). Both sets of coils have similar construction and arrangement, permitting displacement of the beam parallel to the optic axis after it passes through the aperture. Specifically, each assembly of coils consists of two sets of coils lying in different planes; in each plane, four co-planar Helmholtz coils allow the beam to be displaced in the $X$ and $Y$ axes of the coils. The currents applied to these coils are fully user controllable through the parameters Gun (and Aperture) Align $X$ and Align $Y$. It should be noted that above these sets of coils there is a magnetic condenser lens that is activated only in high probe current mode to boost the electron density of the beam. The column also includes stigmator coils for classical corrections of the distortion of the electron beam. However, both of these components are working in the same manner in conventional scanning mode and in the HR-SACP rocking mode presented here, and will be ignored in this discussion.

Under normal imaging conditions (Fig. 1), the objective lens assembly (C3) focuses the beam at the desirable working distance. The objective lens consists of a combined electrostatic/electromagnetic lens triplet to minimize achromatic and spherical aberrations. A single-stage beam scanning system is integrated in the lens, just above the electromagnetic lens gap, resulting in minimization of transverse chromatic and other lenses aberrations. If no beam shift is superimposed on the scanning coils (SC), the beam is scanned symmetrically about the optic axis (Fig. 1a). Note that the focusing action of the objective lens inverts the direction of the beam deflection controlled by the SC. To scan over an area of interest not centered on the optic axis, without moving the sample, an additional beam shift can be applied, as illustrated in Fig. 1b. This beam shift applies an offset to the range of current variation of the SC. The $X$ and $Y$ beam shifts are also user controllable parameters.

\subsection{Detailed principle of the rocking beam mode}

Our rocking beam method is illustrated Fig. 2, starting from the basic concept of ECP collection in conventional scanning mode (Fig. 2a) and progressively showing the way the SACPs are collected in rocking beam mode (Fig. 2d). The scheme of the column shows the beam striking a large grain or single crystal in scanning mode at low magnification in Fig. 2a-c, but striking the sample at a single point in Fig. 2d. The resulting backscattered electron (BSE) images and SACP are represented schematically below each figure. 


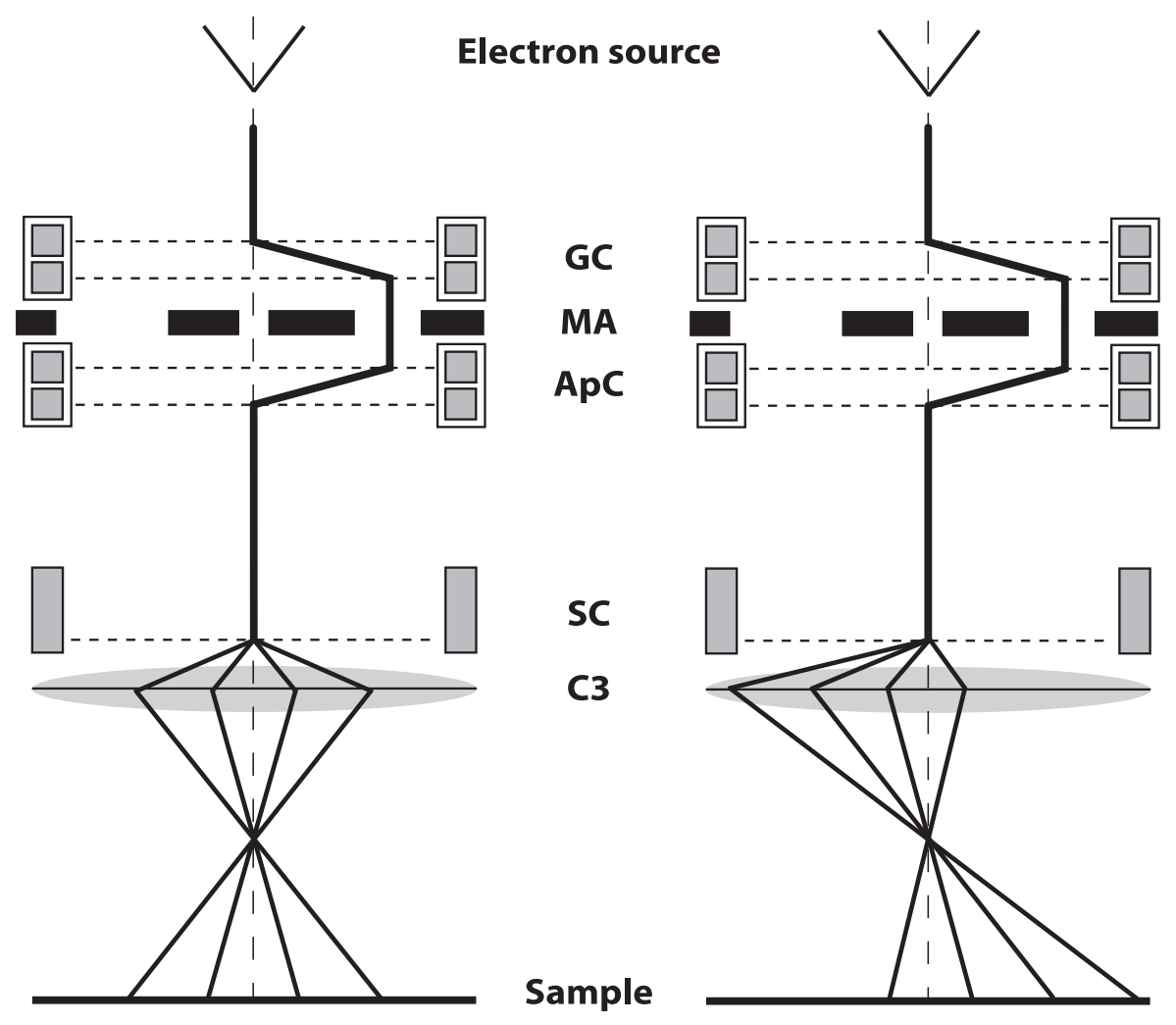

Fig. 1. Simplified scheme of the Gemini column. (a) Electron beam path in scanning mode with off-axis aperture selected using beam deflections above and below the aperture and no beam shift applied at the scanning coils. (b) Electron beam path in scanning mode using an off-axis aperture and with beam shift applied at the scanning coils. GC: gun coils, MA: multihole aperture, ApC: aperture coils, SC: scanning coils, and C3: objective lens. Horizontal dotted lines represent the deflection planes of the various coils. Distances between elements are not representative.
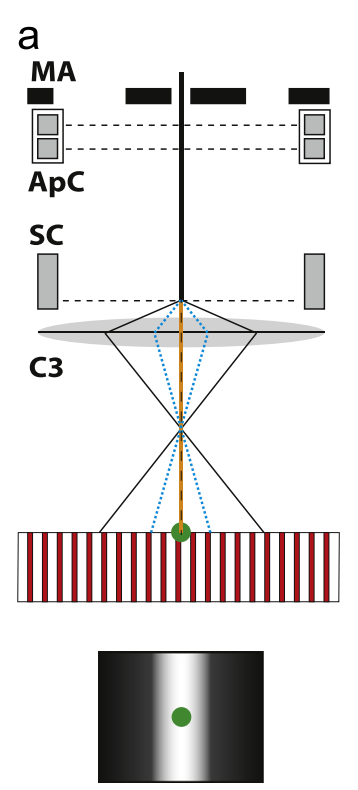

b
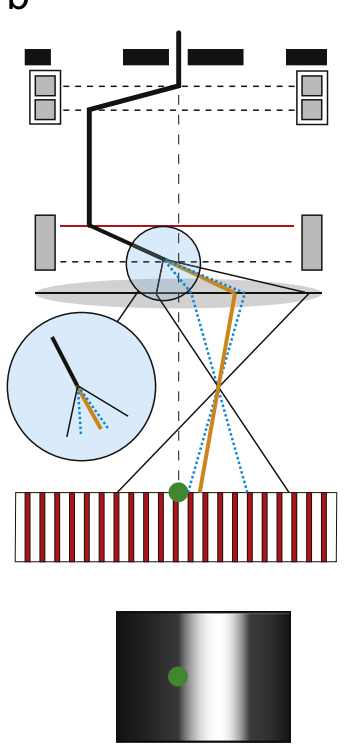

C
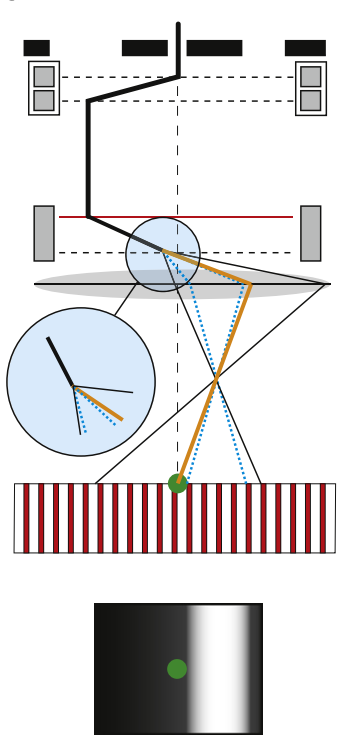

d
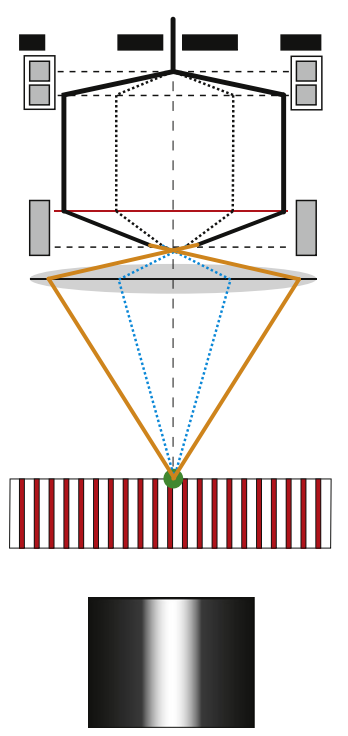

Fig. 2. Principle of the rocking beam method, starting from the basic concept of ECP collection and progressing to the method for SACP collection. Dotted beams in blue are at the Bragg condition with respect to the crystallographic planes shown in red. The orange beam is at the center of the scanning range, referred to as the Spot Beam. The green dot is a surface detail of interest centered on the optic axis. Horizontal dotted lines represent the deflection planes of the coils. (a) ECP acquisition at low magnification. (b) Beam deflection from the ApC resulting in additional deflection from the SC. The beam entering in SC magnetic field is parallel to the optic axis and is deflected by the SC toward the optic axis. This deflection is schematically represented by the horizontal red line. (c) The beam shift control is used to re-center the scanning area about the detail of interest. (d) SACP beam rocking mode resulting from scanning with the ApC and applying beam shift corrections to focus all the beams on the same rocking point. Distances between elements are not representative. Schematic representations of corresponding channeling bands in relation to the detail of interest are shown at the bottom of each figure. (For interpretation of the references to colour in this figure legend, the reader is referred to the web version of this article.) 
The green dot represents a small detail of interest on the sample. The Primary Beam is defined as the electron beam prior to entering the SC and is depicted by heavy black lines. The full width of the scanning area (ECP mode in Fig. 2a-c) is indicated by lighter black lines. Inside this scanning range specific beams are considered: (1) beams at the Bragg condition are shown as dotted blue lines (Bragg Beams) and (2) the beam at the center of the scanning range is shown in orange (Spot Beam).

Fig. 2a shows the microscope configuration in conventional imaging mode, which is also used to collect an ECP from a single crystal or from a large grain (i.e. very low magnification imaging). The beam shift is set to zero, so the Spot Beam is coincident with the optic axis. The beam is scanned over a region centered on the detail of interest, set by stage movement coincident with the optic axis. Thus, C3 focuses the Spot Beam on the detail of interest. Assuming the crystal is oriented such that the Spot Beam is parallel to the crystallographic planes in red, the channeling band will be centered about the detail of interest in the BSE image (illustrated at the bottom of Fig. 2a).

In Fig. $2 b$, the Primary Beam is deflected (to the left) by the ApC. In practice, this process is the result of two coordinated deflections controlled by the ApC through one command. Now the Primary Beam is parallel with the optic axis, but as the Primary Beam is not passing through the center of the SC, it will be deflected toward the optic axis by the magnetic field of the SC (the SC cannot be conveniently deactivated in this procedure, so a residual deflection occurs for off-axis beams). This additional deflection is denoted by the horizontal red line in Fig. 2b. In practice, this deflection and the action of the SC occur at the same location in the microscope, which in the Gemini column is also very close to the location where the objective lens action occurs. However, for illustrative purposes, the schematic representations shown here in Fig. 2b and c illustrate these three actions occurring at different planes. At this point (Fig. 2b), there is no beam shift applied, so the Primary Beam and Spot Beam are still coincident (as shown in blue circular inset in Fig. 2b). The scanned area is shifted (to the right) and is no longer centered about the optic axis of the column. Thus, the objective lens no longer focuses the Spot Beam on the detail of interest and the Bragg Beams are no longer centered about the Spot Beam, but are instead shifted (to the right). This has two consequences on the ECP: the channeling band moves to the right while the detail of interest moves to the left.

So as to rock the beam on the detail of interest, the Spot Beam must be repositioned on the detail, as illustrated in Fig. 2c. In the SACP approach presented here, this is achieved by applying a beam shift that adds an additional deflection to the Spot Beam so that the focusing action of $\mathrm{C} 3$ deflects the Spot Beam back to the detail of interest. This deflection is highlighted in Fig. $2 c$ at the deflection plane of the SC (black dotted line). It is also shown in the blue circular inset in Fig. 2c that the Spot Beam is no longer coincident with the Primary Beam. The Spot Beam now follows the path required for beam rocking: it strikes the detail of interest at an angle. Nevertheless, although the scanned area is again centered on this detail, the Bragg Beams are shifted to the right of the scanning range, and the resulting channeling band is shifted to the right.

Finally, Fig. 2d shows the rocking beam mode for SACP collection. The beam is first deflected from the optic axis by the ApC. The Primary Beam entering the magnetic field of the SC is then deflected toward the optic axis. This deflection is compensated for by beam shift corrections to ensure that, after the focusing action of $\mathrm{C} 3$, the Spot Beam will be centered on the pivot point (the detail of interest). It is worth noting that the SC are no longer used for scanning but instead only used to control the beam shift, while the beam rocking is controlled by the ApC. The collected SACP appears the same as the ECP collected from a single crystal, shown in
Fig. 2a, but the entire SACP is collected only from the detail of interest.

In order for the SACP to be collected from a small, defined area, this rocking beam procedure requires the beam shift correction to be calibrated as a function of the $X$ and $Y$ deflections of the ApC. This calibration file must be generated and stored by the user for a given set of SEM working conditions (accelerating voltage, working distance, and aperture size and position). As demonstrated later, this user-calibrated beam shift correction is the key to the improved spatial resolution of this method.

\subsection{SACP collection as a function of rocking angle}

In this study, the BSE signal to form the SACPs is collected using a standard 4-quadrant pole piece mounted Si-diode detector, as illustrated in Fig. 3. Based on the microscope configuration and functionality described above, the SACPs are collected as outlined in the three steps below:

(a) The beam is first deflected off the optic axis using the $X$ and $Y$ deflection commands of the ApC. These $(X, Y)$ deflections are carried out in a systematic manner, shown by the snake path on the $X-Y$ grid in Fig. 3, in order to minimize the command variations for moving the beam from one position to the next and to minimize latency between command and response. The number of $(X$ and $Y$ ) steps in the deflection range are user controllable, and will influence the acquisition time and the angular resolution of the SACP.

(b) The beam is then deflected by the $X$ and $Y$ beam shift controls of the SC to correct the beam trajectory. As mentioned previously, the beam shift calibration must be available for the selected SEM working conditions.

(c) The SACP signal is collected on the BSE detector. For each $(X, Y)$ deflection of the ApC (starting from the minimum $X$ and $Y$ position), the entire BSE intensity is recorded. This BSE intensity function is progressively displayed to build the SACP with $X$ ApC deflection as the abscissa axis and $Y$ ApC deflection as the ordinate axis.

\section{Application results}

\subsection{Experimental procedure}

The SACP development was conducted on a Crossbeam FEG SEM Zeiss Auriga 40 equipped with the GEMINI-type electron column, the Orsay Physics COBRA ion column, and a gas injection system allowing deposition of platinum. The BSE signal was collected using a 4 quadrant Si diode detector with $11 \mathrm{~mm}^{2}$ surface area. Automation was done through the Zeiss API version 2.9 communication tool. All of the experiments were carried out with a working distance of $10 \mathrm{~mm}$, a high tension of $20 \mathrm{kV}$, and an aperture size of $30 \mu \mathrm{m}$ (the center aperture).

The beam shift corrections have been calibrated manually for more than $200(X, Y)$ ApC deflections. Before starting the calibration, lens hysteresis corrections, column alignments, and astigmatism corrections were performed with the ApC and SC set to zero. The beam shift calibrations were carried out in imaging mode by centering a detail of interest at the center of the screen. For each $(X, Y)$ beam deflection command by the ApC, the $(X, Y)$ beam shift correction necessary to bring the detail of interest back to the screen center was recorded. It should be noted that the $X-Y$ axes of the aperture coils are not parallel to the respective $X-Y$ axes of the scanning coils. Therefore, an $X$ ApC deflection command must be compensated by both $X$ and $Y$ beam shifts. All the 


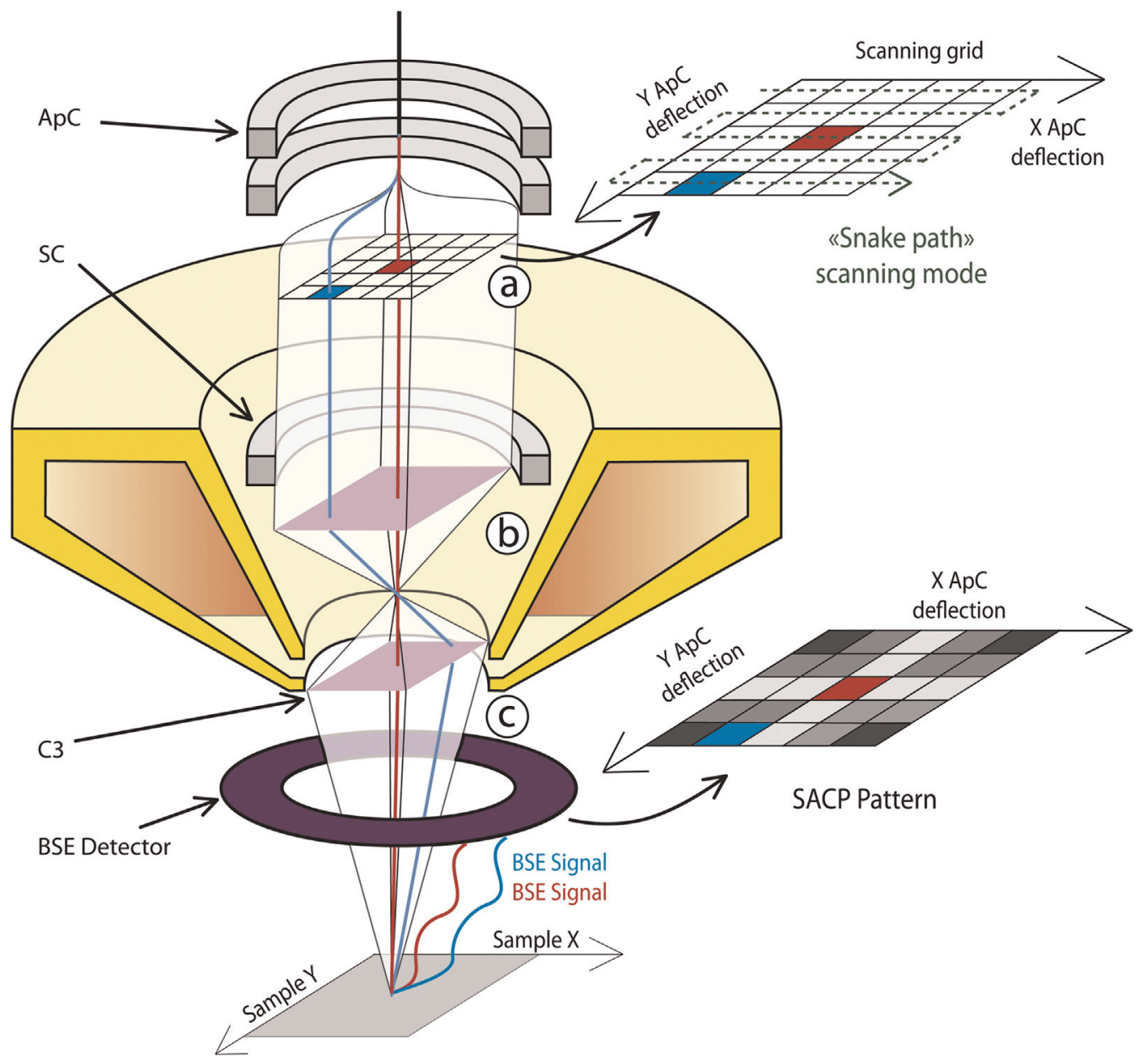

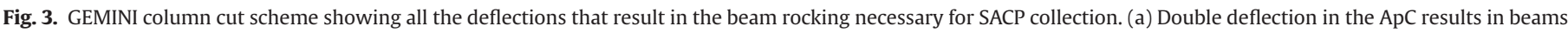

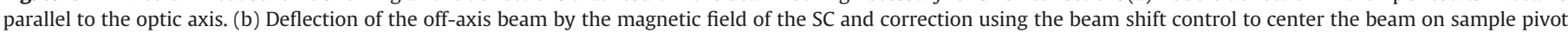

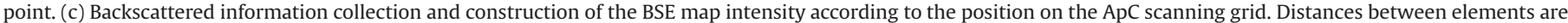
not representative.

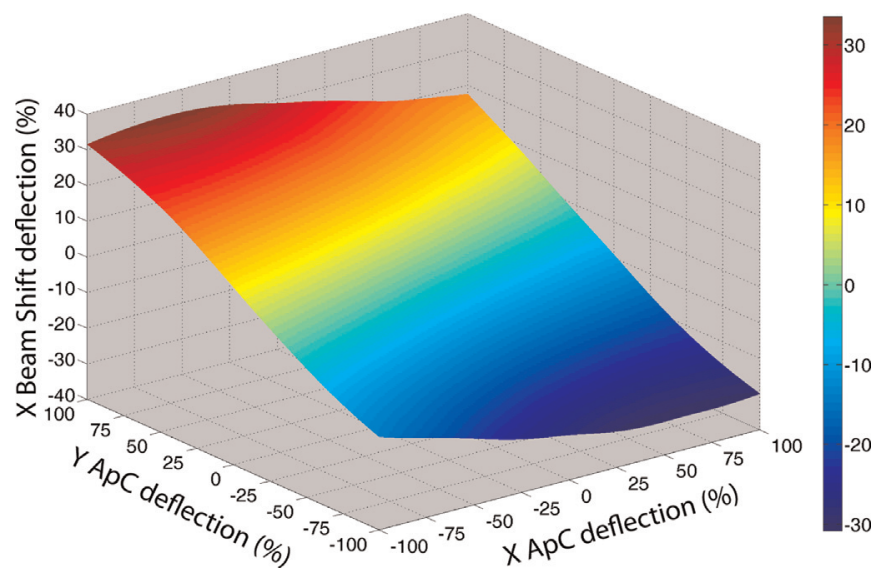

Fig. 4. $X$ beam shift calibration as a function of $X$ and $Y$ ApC deflections.

200 points were recorded during the same session without switching off the high voltage. From these data, a calibration surface mesh was fitted to predict all of the $(X, Y)$ beam shift corrections for each $(X, Y)$ ApC deflection. Fig. 4 shows an example $X$ beam shift calibration curve as a function of $(X, Y)$ ApC deflection (the corresponding $Y$ beam shift calibration is not shown).

In this paper, the collection of High Resolution SACPs is illustrated on a [001] single crystal of silicon. The default sampling size for the high resolution SACPs (HR-SACPs) is $100 \times 100$ pixels, which takes about four and a half minutes to collect. To facilitate SACP recognition, the patterns were compared with diffraction patterns simulated from orientation data determined by EBSD. These EBSD experiments were carried out by tilting the sample stage $70^{\circ}$ and collecting EBSD patterns using a Bruker Quantax CrystAlign system.

\subsection{Characteristic features of HR-SACPS}

\subsubsection{Typical HR-SACP collected on the Gemini type column}

Fig. 5 shows a typical HR-SACP (Fig. 5b) obtained with the procedure outline above and compares it with a conventional ECP (Fig. 5a). The SACP image quality is comparable to that of the ECP despite the difference in image resolution: the ECP was collected with conventional SEM resolution $(1024 \times 768$ pixels) with an angular range of $\sim 7^{\circ}$ while the HR-SACP contains $100 \times 100$ pixels covering an angular range of $\sim 4^{\circ}$ (gray levels in both raw images are identical and no post acquisition treatment was done).

Because the ECP is collected in conventional imaging mode, the linear scale shown in the image is accurate, reflecting that the ECP was collected from an area approximately $3.8 \times 2.8 \mathrm{~mm}^{2}$ in size. Black points in the ECP result from surface defects in this area of the Si single crystal. These defects observed in the ECP are absent on the HR-SACP, as shown in the correlation image (Fig. 5c) the 
a

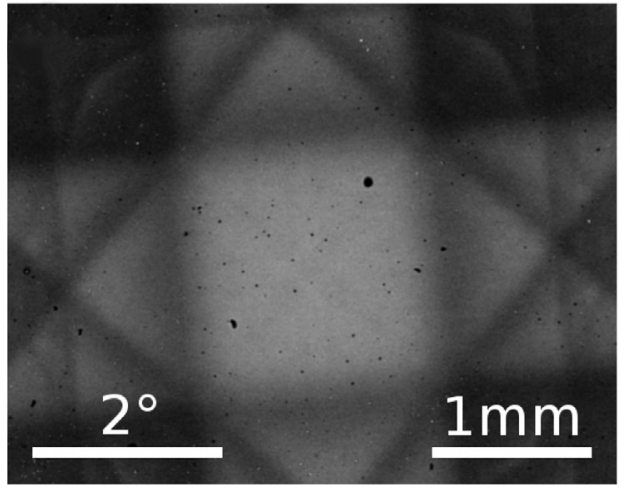

b

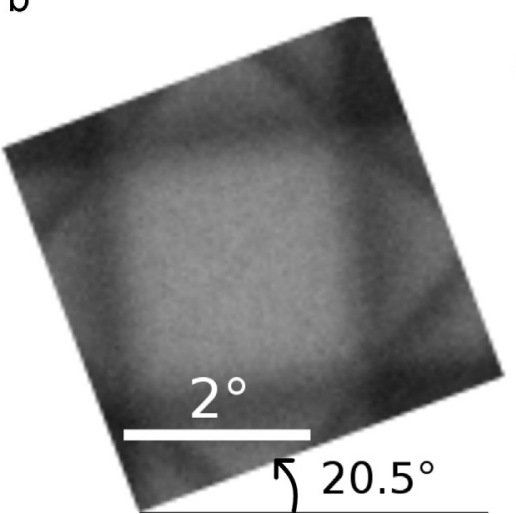

C

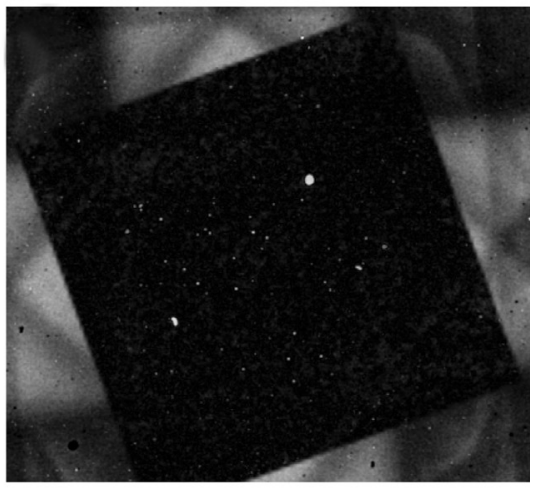

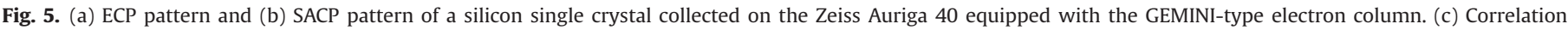

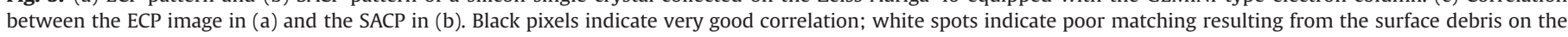
$\mathrm{ECP}$, as seen in (a).

HR-SACP pattern was collected from a significantly smaller area, free of defects.

The HR-SACP has been rotated to overlap the ECP in Fig. 5. The need for this rotation correction stems from two factors. It should be recalled that the bands observed in an ECP are parallel to the planes responsible for their formation i.e. there is no rotation of an $\mathrm{ECP}$ relative to the scanned image. But there is an inherent $180^{\circ}$ rotation (inversion) between the ECP and the HR-SACP. The reason for this is seen in Fig. 2, which shows that the beam incidence angle is reversed between the ECP formation (Fig. 2a) and the HRSACP formation (Fig. 2d) i.e. beams scanned to the left strike the sample with a positive angle in the ECP, but with a negative angle in the HR-SACP. An additional rotation occurs for the HR-SACP because the deflection axis of the ApC and the SC are misaligned (i.e. the $X$ deflection of the ApC is not parallel with the $X$-shift of the SC). On the particular microscope used in this study, this misalignment has been measured as $-20.5^{\circ}$. Combining these two rotations results in a $180^{\circ}-20.5^{\circ}=159.5^{\circ}$ rotation between the HR-SACP and the true crystallographic orientation of the specimen. Validation of this rotation angle has been carried out on various orientations where results are not ambiguous as a result of pattern symmetry (i.e. using zone axes that do not have rotational symmetry).

\subsubsection{HR-SACP angular range}

Fig. 5 also reveals that the collected HR-SACPs exhibit an angular range of more than $4^{\circ}$, slightly smaller than that obtained from the ECPs. The calculation was performed from the known angular width of the observed (220) bands, assuming angles vary linearly with distance on the images (which is true for small angles). While this range is rather small, it is still large enough to distinguish a few bands that can easily be indexed from a simulated EBSD pattern, as demonstrated in Section 4 and in [35].

The angular range of the HR-SACP is directly linked to the range of the $X$ and $Y$ deflections controlled by the ApC. This angular range was measured with a precision of $0.1^{\circ}$ on ECPs with $1024 \times 768$ pixel resolution. Two high resolution ECPs were collected respectively with the ApC $X$ deflection set to its minimum and maximum values (with the required beam shift corrections to center the scan on the optic axis and with the $Y$ deflection set to $0)$. The same was done for the two extreme $Y$ deflections. The electron path in the Gemini-column for such ECP collection is illustrated in Fig. 2c. The shift of the diffraction band induced by the deflections of the ApC can be seen in Fig. $2 c$ in comparison to a conventional ECP configuration in Fig. 2a. The shift induced by the two extreme $X$ deflections was used to precisely measure the corresponding angular range possible with the ApC. a

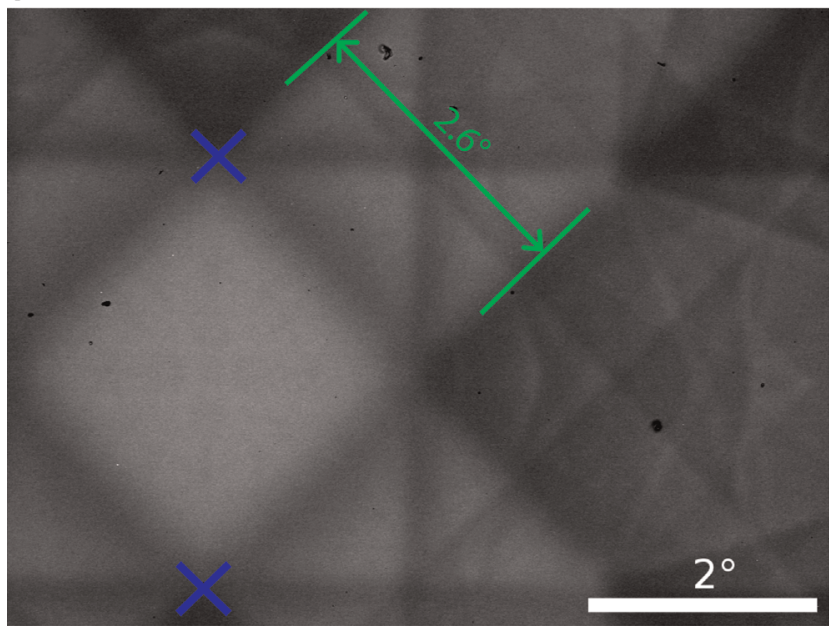

b

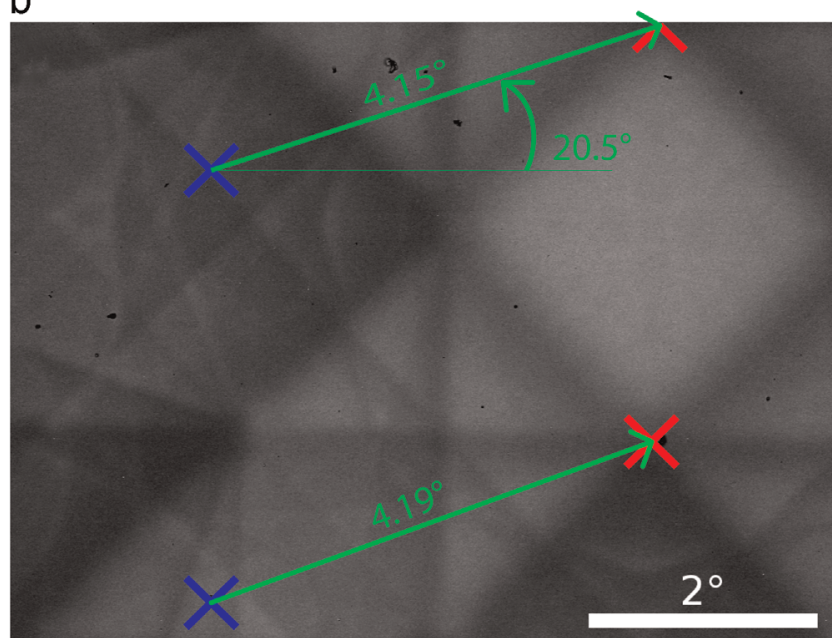

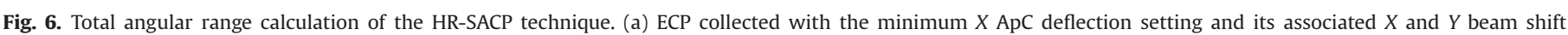

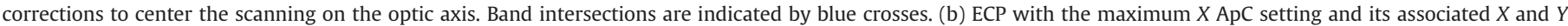

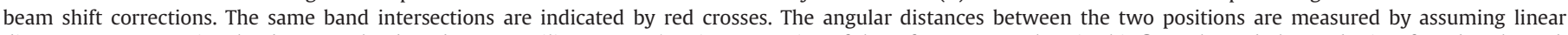

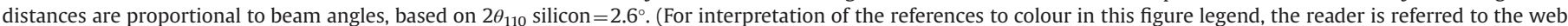
version of this article.) 
Fig. 6 shows the resulting ECPs, with $1024 \times 768$ pixel resolution, for the two extremes in the $X$ deflection. The blue crosses indicate the band intersections in the ECP collected for the minimum $X$ deflection of the ApC, while the red crosses indicate the same band intersections in the ECP collected with the maximum $X$ deflection of the ApC. The shift between the two ECPs is shown by the green lines in Fig. $6 b$ (note that the defects in the Si single crystal have not moved on the images, but the channeling bands have moved). This shift corresponds to the total $X$-width possible for our HR-SACPs. The $X$ and $Y$ extreme deflections provide for an angular range of $4.2^{\circ}$ for our HR-SACPs. It is also useful to note that the lines connecting the red and blue crosses inn Fig. $6 \mathrm{~b}$ lie at $20.5^{\circ}$, indicative of the misalignment of the $X-A p C$ deflection and $X$-SC deflection that results in the rotational part of the misalignment between ECP and SACP discussed above.

\subsubsection{HR-SACP spatial resolution}

The spatial resolution of SACP is defined as the smallest area from which the backscattered information is collected. While it is readily accepted that SACPs offer spatial resolution improvements over ECP, the actual spatial resolutions that can be achieved with different approaches for collecting SACP have typically not been demonstrated.

In this paper, a methodology to accurately measure the spatial resolution has been developed. Using the FIB in conjunction with gas injection, polycrystalline platinum squares of different sizes were deposited on the single crystal silicon. Due to the difference in the atomic number $Z$ between Pt and $\mathrm{Si}$, a large variation in BSE signal intensity is expected when the beam strikes the Pt or Si. To increase this $Z$-contrast, the BSE image contrast was maximized so that the intensity was maximum when the beam strikes Pt (gray scale of 255) and minimum (gray scale of 0 ) when the beam strikes Si. Thus when the beam is centered on a Pt square and rocking mode is applied, the pixels in the resulting output will have maximum intensity when the beam falls on the platinum, but minimum intensity when the beam falls outside the Pt square. This makes it very easy to quantify the percentage of information coming from the square versus the amount of information that comes from outside the square. It is worth mentioning that no SACP pattern is expected when the beam is rocking over the Pt squares as they are not single crystal.

Fig. 7a illustrates the series of platinum squares used to demonstrate the spatial resolution of our rocking beam approach. Fig. $7 \mathrm{~b}$ and $\mathrm{c}$ shows the contrasted BSE images collected in rocking

a

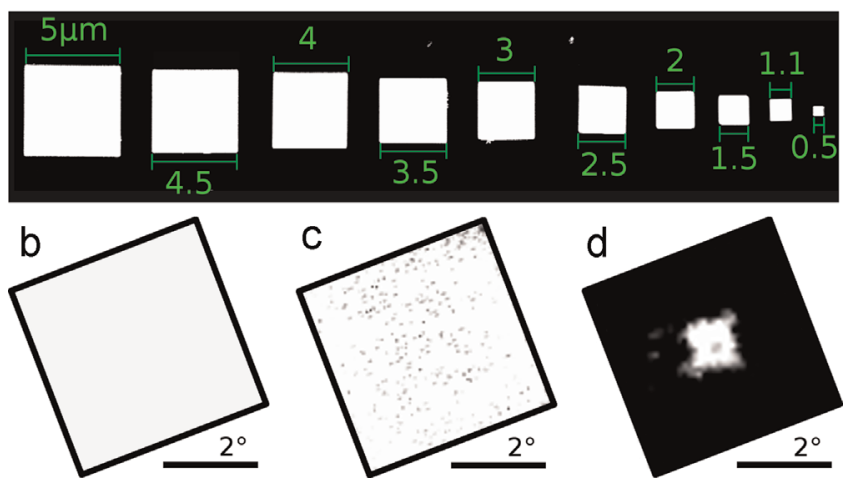

Fig. 7. Illustration of the spatial resolution of the HR-SACP technique. (a) BSE image of deposited platinum squares on silicon. (b) HR-SACP rocking beam mode output from the $1.1 \mu \mathrm{m}$ Pt square. (c) HR-SACP rocking beam output from the $500 \mathrm{~nm} \mathrm{Pt}$ square. (d) Illustration of the degradation of the spatial resolution resulting from poor beam alignment, focus, and without hysteresis corrections, for an HR-SACP collected from the $500 \mathrm{~nm}$ Pt square. Black contouring on (b) and (c) is added after acquisition to distinguish the edges of the output. mode with the beam centered on the $1.1 \mu \mathrm{m}$ and $500 \mathrm{~nm}$ platinum squares respectively. In Fig. $7 \mathrm{~b}, 100 \%$ of the pixels display maximum intensity (level of 255), indicative that all of the rocked beams fell upon the $1.1 \mu \mathrm{m}$ Pt square. In Fig. 7c, the pixels with maximum intensity drop to $82 \%$, indicative that some rocked beam fell outside the 500nm square. However, if pixels in the upper half of the contrast spectrum (i.e. 128-255) are considered, all the pixels can be attributed to the $500 \mathrm{~nm}$ square. This demonstrates that the spatial resolution of our approach is below $500 \mathrm{~nm}$ at the working distance of $10 \mathrm{~mm}$. However, to achieve this spatial resolution, careful beam alignment is required. Fig. $7 \mathrm{~d}$ shows a rocking beam image from the same $500 \mathrm{~nm}$ square prior to careful beam alignment, focus, and lens hysteresis correction. The percentage of pixels intensities at 255 and in the range 128-255 drops to $3.4 \%$ and $6.2 \%$ respectively.

\subsubsection{Angular accuracy and precision of the HR-SACP to set the channeling conditions}

The critical aspect of the precision and accuracy of the HRSACPs developed here is that they allow unambiguously the setting up of channeling conditions for ECCI, including the ability to accurately determine not only the specific $g$, but also the deviation from the Bragg condition.

In this section, we evaluate the angular accuracy of our HRSACPs with respect to this function. The purpose is not to define how accurate the full orientation matrix can be determined with respect to a reference frame (as in case of the EBSD technique), but instead how accurate the crystal zone axis relative to the beam can be determined. This accuracy of SACPs has generally been accepted to be about $0.1^{\circ}[10]$. Here we show that this limit is easily reached by the HR-SACP approach developed in this work.

In practice, the accuracy is derived from how closely the unrocked beam and a specific band edge can be located in the SACP. With a properly aligned microscope, the un-rocked beam is at the center of the SACP. We determined that repeating beam alignments always resulted in the same un-rocked beam position relative to the channeling bands in the SACP, at least at the pixel resolution of the $100 \times 100$ SACP (the angular range of each pixel is $0.042^{\circ}$, based on the $4.2^{\circ}$ angular range of the patterns). This confirms that the beam is aligned with an accuracy and a precision better than $0.04^{\circ}$.

Typically the edge of a channeling band cannot be detected within the pixel resolution of our reference SACP. Each band edge has a specific width inherent to the channeling phenomenon itself. Moreover, additional instrumental and material limitations, including energy spread in the incident electron beam and crystal defects, will also result in channeling band broadening. We estimate that band edge covers an average of 4 pixels of an SACP of $100 \times 100$ pixels (that means an angular range of $0.17^{\circ}$ ). Thus the middle of an edge relative to the beam can be defined with an accuracy better than $0.1^{\circ}$.

The un-rocked beam position determined from the HR-SACP is finally compared to the incident beam position deduced from EBSD orientation measurements. Fig. 8a shows an HR-SACP collected at $0^{\circ}$ tilt on the Si sample, which shows a $\langle 001\rangle$ zone axis less than $0.2^{\circ}$ from the optic axis (represented by blue cross). An EBSD pattern of the same sample area was also collected, but at the typical $70^{\circ}$ stage tilt, and the resulting orientation (with respect to the sample coordinate system through the stage reference frame) was used to predict the corresponding $0^{\circ}$ tilt EBSD/channeling pattern. This simulated EBSD pattern at $0^{\circ}$ tilt (Fig. $8 \mathrm{~b}$ ) indicates that the $\langle 001\rangle$ zone axis is shifted by $\sim 2^{\circ}$ from the optic axis (represented by red cross). This EBSD experiment was repeated a total of eight times, resulting in an average difference of $1.8^{\circ}$ between the EBSD predicted incident beam position and that determined from the HR-SACP. This error is most likely a result of 

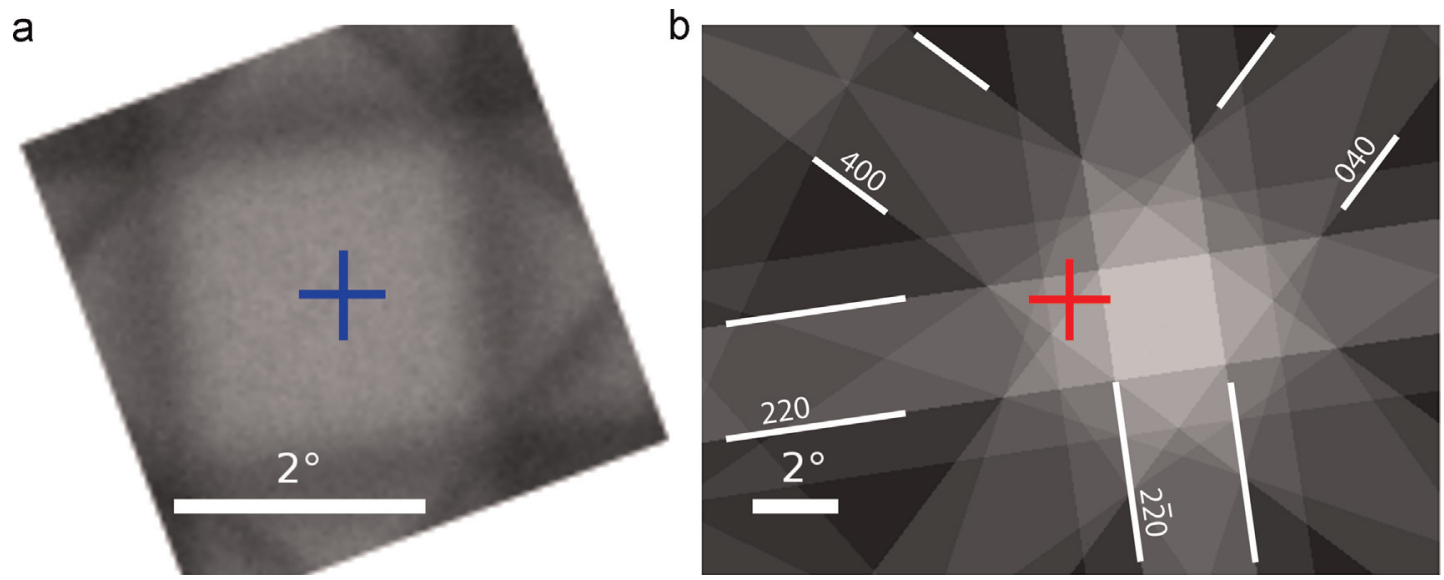

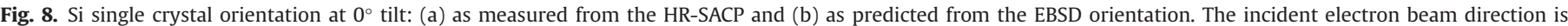

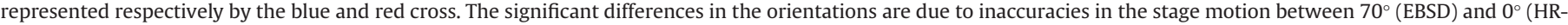

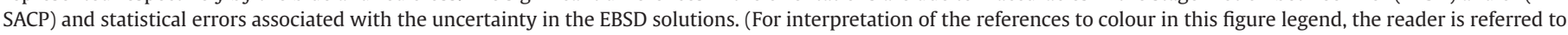
the web version of this article.)

both systematic errors, such as those associated with stage tilt calibrations, and statistical errors associated with the uncertainty of the EBSD solutions [27].

Because the SACP directly reveals the orientation of the crystal with respect of the optic axis, it is advantageous over EBSD for accurately setting up channeling conditions for A-ECCI. The simulated pattern from the approximate EBSD orientation is nevertheless useful for indexing the HR-SACP, which has limited angular range.

\section{Discussion}

The results presented here demonstrate two critical highlights. First, the SACP beam rocking procedure has been developed on a FEG Gemini column, the first demonstration of SACP collection on this column. Secondly, an efficient method to collect SACPs from $500 \mathrm{~nm}$ areas at $\mathrm{WD}=10 \mathrm{~mm}$ has been developed, justifying the term "High Resolution SACP".

\subsection{Innovative procedure to collect SACP on the Gemini column}

In order to effectively rock the electron beam on the sample surface at a reasonable working distance, the electron beam should be deflected at two locations in the column. Different approaches have been proposed to carry out these coordinated beam deflections in an SEM. The first approach, developed by Van Essen and Schulson [7] and known as the double deflection method, is achieved by controlling the deflection of the second set of coils involved in the scanning stage so as to deflect all the beams on the same point. Consequently, the beam does not pass through the intersection of the objective lens plane with the optic axis. The objective lens is then deactivated to avoid a further deflection. The focusing of the beam on the sample is instead carried out by the final condenser lens (last lens before the objective lens). The main drawback of this approach is the poor spatial resolution. Indeed, the two scanning coils used in this approach are typically electronically synced. Reducing the deflection of the second scanning stage requires the second coil assembly to be modified with a variable resistor, which is adjusted to move tilted beams to establish the rocking point. However, this variation in resistance, which is fixed for a given SACP condition, is not expected to be the same for all tilted beams, and consequently the approach results in a significant area of confusion. Moreover, as the focusing of the beam in this approach is carried out by a condenser lens that is not designed for imaging, large aberrations of the beam, which occur when it is tilted off axis, contribute to a decrease in the spatial resolution. With these two effects, resulting spatial resolution of this double deflection approach was approximately $50 \mu \mathrm{m}$ [7].

To overcome these problems, a second technique, called the deflection focusing method, was then developed by Van Essen et al. [8]. In this technique the second scanning coil assembly is shut off and the second beam deflection is accomplished by the objective lens. Both of the limitations of the double deflection approach were reduced, and the improved spatial resolution was evaluated to be $10 \mu \mathrm{m}$. This deflection focusing technique was adapted for the SACP mode most commonly used in commercial SEMs. Hall et al. [36] further improved the spatial resolution of SACPs by developing a spiral scanning approach, which resulted in reduced latency and hysteresis in the response of the objective lens. The major problem of the deflection focusing technique resides in the difficulty in switching between imaging mode and rocking beam mode. It requires both the objective and final condenser lenses to be manually varied between imaging and beam rocking, which involves having to overcome significant hysteresis in both lenses [10].

Currently, only Tescan provides a commercially available SEM rocking mode, which uses an approach that is significantly different from those outlined above [11]. Two scanning stages are used to shift the scanned beam parallel to, but off the optic axis of the objective lens. This beam is then focused to a rocking point on the sample surface by the objective lens. Spherical aberration correction is provided to improve the SACP spatial resolution. However, this approach is not entirely detailed in [11].

The approach illustrated in Fig. $2 \mathrm{c}$ and $\mathrm{d}$ is distinct from the methods mentioned above in two ways. First, it consists of a more complex series of deflections. Two defections are performed at the ApC plane to shift the beam off the optic axis (although only one command is sent to this coil system). An additional deflection is carried out by the SC to correct the deflection occurring when offaxis beams enter the SC. The focusing action of the objective lens results in the final deflection to the rocking point (Fig. 2d). Secondly, the key aspect that enables the improved spatial resolution is the user-developed calibration, used to superimpose an additional beam shift on the scanning coils to optimize the rocking pivot point on the sample surface. This new approach results in superior spatial resolution and is not limited by hysteresis problems in switching from imaging to rocking beam mode, as the lens excitation remains the same for both conditions. 
The need for multiple deflections is more a consequence of the Gemini column configuration, detailed in Section 2.1, than an advantage to the beam rocking method. Indeed, only one set of scanning coils is available on the Gemini column, but because the microscope uses a fixed, electronically adjustable aperture, rather than a traditional mechanically adjusted aperture, additional coils $(\mathrm{ApC})$, designed for large beam deflections to the various apertures, can be used to deflect the beam. Nevertheless, the application programming interface (API) of the instrument limits the ApC deflections such that the resulting SACP angular range is limited to $4.2^{\circ}$, as demonstrated in Section 3.2. Larger beam deflections are possible with the available coils, but our experience shows the SACP begins to be limited by the column bore or other fixed apertures or detectors in the column.

Digital calibrations have never been developed for instruments with commercially integrated rocking beam modes. In the early development of SACP such precise digital control was clearly not available on the analog instrument at hand. Some instruments integrated corrections, such as dynamic focusing of the objective lens to overcome spherical aberration (CamScan microscopes). However, such an approach was carried out using electronic gain controls ( $\gamma$ curves), which are not as precise as digital calibrations. Such corrections also suffer from significant latency and hysteresis limitations in the objective lens.

In the approach presented here, the objective lens excitation is not modified, so latency and hysteresis are not a problem. Furthermore, the digital calibration is carried out for specific microscope operating conditions, leading to high accuracy corrections in comparison to electronic gain. These corrections account for all microscope effects that may shift the beam, rather than depending on predictive models for specific effects such as spherical aberration. Also, because the calibration can be carried out for essentially any operating condition, including working distance and accelerating voltage, this technique offers flexibility to collect SACPs under any conditions that allow a variety of stages and/or detector configurations. This approach to improving resolution is not limited to the Gemini column, but can be implemented on any microscope once a beam rocking procedure is implemented.

While this new method has a significant spatial resolution advantage, it is limited in the need to perform the calibration, which takes about 5 min. Furthermore, the collection of a given SACP takes approximately four and a half minutes, during which time contamination of the area of interest may occur. This relatively slow collection time results in part from the communication system of the API control interface. It may be possible to improve this acquisition time by a commercial hardware control approach.

Alternative methods to carry out SACP were also tested on the Gemini column. For example, once the ApC were used to deflect the beam, the scanning coils were switched off and any beam parallel to the optic axis should be focused at the pivot point (following the approach of Dluhoš et al. [11]). In this case the beam rocking should occur directly without any action of the scanning coils. However, the ApC deflection does not necessarily bring all deflected beams parallel to the optic axis, which would result in beams being shifted from the rocking point. Furthermore, the API interface does not provide a command to allow the scanning coils to be turned off, to do so would require a hardware modification. Another possible approach to carrying out SACP on the Gemini column would be to use an existing beam cross-over to act as a rocking point. Fig. 1a shows that the beam path on the Gemini column for normal imaging mode scanning results in a scanning cross-over that might be used as a pivot point. However, it was determined that this cross-over is located well inside the objective lens and cannot serve as an effective rocking point.

\subsection{Robust method to demonstrate the HR-SACP spatial resolution}

It should be noted that while many papers that deal with SACP have indicated and/or speculated about spatial resolution, in most cases these resolutions have not been clearly demonstrated. In most studies, this resolution has been evaluated by collecting patterns from grains of a given size. If during rocking, the beam leaves the grain of interest, additional noise or distorted bands from grains outside the pattern are observed. However, it is not always easy to clearly identify this additional noise from regions outside the area of interest. Consequently, it is difficult to directly quantify the spatial resolution with this approach.

In this paper, a robust method for demonstrating the spatial resolution of SACPs is presented, which uses calibrated squares of platinum deposited on silicon to determine if the beams forming the SACP fall within or outside of the area of interest. Due to the difference in the atomic numbers of Pt and Si, the strong differences in backscattered yield result in the ability to unambiguously quantify the number of beams that fall within the rocking beam area (as illustrated in Fig. 7). It should be noted that Pt squares were used for this calibration rather than circles due to the difficulty in accurately depositing circular shapes with ion beam assisted deposition. For the same reason, squares smaller than $500 \mathrm{~nm}$ were not studied. As currently demonstrated, nearly all of the rocking beams fall within the defined $500 \mathrm{~nm}$ Pt square. It is possible that by using finer features to carry out the calibration or if a more time consuming calibration were carried out, the spatial resolution could be improved significantly. As the calibration is only limited by the ability to return deflected beams to the area of interest, the calibration is only limited to the microscope beam positioning precision, which is in the range of a few nanometers. Given this, it is quite reasonable to expect SACP spatial resolutions well below $100 \mathrm{~nm}$ to be possible, approaching the limitations imposed by the BSE/channeling interaction volume. There is one potential additional issue that might limit the ability to reach such high spatial resolutions. This is the beam astigmatism that will result from the beam being rocked to large angles. That is, the beam convergence angle, which is symmetric about the optic axis when the beam is not tilted, will result in the two extremes of the beam convergence being subject to different degrees of spherical aberration in the final lens. This will effectively cause the probe to be stretched (stigmatic) at high deflections. It is not clear if this beam spreading will be as large as the spatial resolution limited by other microscope factors. However, high quality objective lenses, such as the achromatic lens in the Gemini column, may reduce this effect. The low beam divergence offered by the Gemini column and many other field emission sources and columns will also serve to minimize this effect.

\subsection{Key features of HR-SACPs for ECCI applications}

The HR-SACP approach presented here is ideal for carrying out quantitative $\mathrm{ECCI}$, because this imaging technique requires the crystal to be accurately oriented to specific Bragg channeling conditions with respect to the incident electron beam. Proper alignment of the imaging system and beam shift control results in the un-tilted electron beam, coincident with the optic axis, to fall at the center of the image. As a result, in HR-SACP mode the pattern center lies at the actual center of the HR-SACP and corresponds to the orientation of the beam relative to the crystal under normal imaging mode. Consequently, the ECC imaging conditions can be directly deduced from the HR-SACP. Without an SACP, it is much more difficult to accurately set up imaging conditions solely from EBSD based orientation information as illustrated in Fig. 8. Thus, this development of HR-SACP greatly facilitates ECCI studies. 
The improved spatial resolution obtained on the Gemini column results from 4 main factors: the rocking angle has a limited range of $\sim 4^{\circ}$, the user-controlled calibration, the recently developed high resolution lenses and the very small beam convergence offered by many field emission gun columns. While a number of early studies showed that SACP spatial resolutions close to $1 \mu \mathrm{m}$ could be obtained, in most commercial SACP modes the resolution was typically much worse than this, often one or two orders of magnitude larger. Furthermore, the optimum spatial resolution was often obtained at impractically small or large working distances that limited the ability to carry out reasonable stage manipulations or led to difficulties with other microscope parameters necessary for ECCI studies [14]. For example, Joy et al. [37] obtained SACPs from an area of $1 \mu \mathrm{m}$ with a total rocking angle of $9^{\circ}$, but at working distance of $1 \mathrm{~mm}$. At such short working distances tilts necessary for setting up ECCI condition often cannot be carried out as they jeopardize the objective lens and/or BSE detector.

In the intervening years, improvements in microscope column design has lead to vast improvement in secondary and backscattered electron imaging resolution. These changes make comparison of SACP capabilities difficult. Furthermore, it is quite uncommon now for microscopes to offer the ability to carry out SACP. As noted earlier, as far as the authors are aware, the only commercially available SACP system is on the Tescan Mira instrument, which has a demonstrated SACP resolution on grains of $20 \mu \mathrm{m}$ with an angular range of $\sim 20^{\circ}$ [11]. This large angular range is consistent with the objective to include a significant number of channeling bands in the pattern to make orientation determination straightforward. However, large SACP angular range comes at a cost of spatial resolution. We have illustrated that such large ranges are not required to solve patterns if simulated patterns, typically available from EBSD system, are available [35].

The enhanced spatial resolution of HR-SACP offers significant advantages for the application of ECCI. Clearly, in order to study crystal defects in small grains, it is necessary to be able to collect the SACP from within the grain. In addition, in many deformation studies of even large grained materials, the crystal distortions resulting from the accumulation of geometrically necessary dislocations makes it impossible to collect SACPs over large areas, as the channeling bands become distorted and blurred. Being able to reduce the area of collection minimizes these effects. Overall, the development of the HR-SACP presented here has important implications on the application of ECCI to broader materials studies.

\section{Conclusion}

For the first time the collection of selected area electron channeling patters (SACPs) from sub-micron sized areas has been demonstrated, with a spatial resolution of $500 \mathrm{~nm}$ at a practical working distance of $10 \mathrm{~mm}$. The key to collecting these high resolution SACPs (HR-SACPs) is to accurately adjust the rocking electron beam position using a digitally calibrated beam shift. The high resolution of these patterns has been demonstrated with a robust method that unambiguously determines the percentage of rocked beams that fall in the area of interest. It is also demonstrated that with an SACP rocking angle of $4.2^{\circ}$ and with accuracies better than $0.1^{\circ}$, these patterns contain sufficient channeling bands to allow accurate indexing in conjunction with simulated patterns. These characteristics make the technique suitable for setting up imaging conditions for Accurate ECCI (A-ECCI) on very fine-grained samples or materials that have significant crystal distortions associated with large amounts of plastic strain. The reasonable $10 \mathrm{~mm}$ working distance allows flexibility for stage movements, allowing a large variety of channeling conditions to be reached for contrast analysis, as well as for the ability to carry out SACP analysis in conjunction with complicated in-situ testing stages. Moreover, this innovative approach offers insights for developing HR-SACP modes on new generation electron columns.

\section{Acknowledgment}

The authors would like to acknowledge the University of Lorraine for the financial support of invited Professor M. A. Crimp.

\section{References}

[1] D.G. Coates, Kikuchi-like reflection patterns obtained with the scanning electron microscope, Philos. Mag. 16 (1967) 1179-1184.

[2] G.R. Booker, A.M.B. Shaw, M.J. Whelan, P.B. Hirsch, Some comments on the interpretation of the 'kikuchi-like reflection patterns' observed by scanning electron microscopy, Philos. Mag. 16 (1967) 1185-1191.

[3] G.E. Lloyd, Atomic number and crystallographic contrast images with the SEM: a review of backscattered electron techniques, Mineral. Mag. 51 (1987) 3-19.

[4] R. Vincent, P.A. Midgley, Double conical beam-rocking system for measurement of integrated electron diffraction intensities, Ultramicroscopy 53 (1994) 271-282.

[5] Proceedings of the electron crystallography school 2005, ELCRYST 2005: new frontiers in electron crystallography, Ultramicroscopy 107 (2007) 431-558.

[6] C.S. Own, W. Sinkler, L.D. Marks, Rapid structure determination of a metal oxide from pseudo-kinematical electron diffraction data, Ultramicroscopy 106 (2006) 114-122.

[7] C.G. Van Essen, E.M. Schulson, Selected area channelling patterns in the scanning electron microscope, J. Mater. Sci. 4 (1969) 336-339.

[8] C.G. Van Essen, E.M. Schulson, R.H. Donaghay, Electron channelling patterns from small (10 [micro]m) selected areas in the scanning electron microscope, Nature 225 (1970) 847-848.

[9] D.C. Joy, G.R. Booker, Simultaneous display of micrograph and selected-area channelling pattern using the scanning electron microscope, J. Phys. E: Sci. Instrum. 4 (1971) 837.

[10] D.C. Joy, D.E. Newbury, D.L. Davidson, Electron channeling patterns in the scanning electron microscope, J. Appl. Phys. 53 (1982) R81-R122.

[11] J. Dluhoš, L. Sedláček, J. Man, Proceedings of the 21st International Conference on Metallurgy and Materials, Brno, Czech Republic, EU, 2012.

[12] J.A. Venables, C.J. Harland, Electron back-scattering patterns-a new technique for obtaining crystallographic information in the scanning electron microscope, Philos. Mag. 27 (1973) 1193-1200.

[13] S.I. Wright, B.L. Adams, Automatic analysis of electron backscatter diffraction patterns, Metall. Trans. A 23 (1992) 759-767.

[14] A.J. Wilkinson, P.B. Hirsch, Electron diffraction based techniques in scanning electron microscopy of bulk materials, Micron 28 (1997) 279-308.

[15] S.I. Wright, M.M. Nowell, D.P. Field, A review of strain analysis using electron backscatter diffraction, Microsc. Microanal. 17 (2011) 316-329.

[16] T. Maitland, S. Sitzman, Backscattering detector and EBSD in nanomaterials characterization, in: W. Zhou, Z. Wang (Eds.), Scanning Microscopy for Nanotechnology, Springer, New York, 2007, pp. 41-75.

[17] M.A. Crimp, Scanning electron microscopy imaging of dislocations in bulk materials, using electron channeling contrast, Microsc. Res. Tech. 69 (2006) 374-381.

[18] A. Weidner, F. Pyczak, H. Biermann, Scanning and transmission electron microscopy investigations of defect arrangements in a two-phase $\gamma$-TiAl alloy, Mater. Sci. Eng.: A 571 (2013) 49-56.

[19] A. Barnoush, Correlation between dislocation density and nanomechanical response during nanoindentation, Acta Mater. 60 (2012) 1268-1277.

[20] S. Primig, H. Leitner, W. Knabl, A. Lorich, H. ClemensR. Stickler, Influence of the heating rate on the recrystallization behavior of molybdenum, Mater. Sci. Eng. A 535 (2012) 316-324.

[21] J. Ahmed, S.G. Roberts, A.J. Wilkinson, Characterizing dislocation structure evolution during cyclic deformation using electron channelling contras imaging, Philos. Mag. 86 (2006) 4965-4981.

[22] Y.N. Picard, R. Kamaladasa, M. De Graef, N.T. Nuhfer, W.J. Mershon, T. Owens, L. Sedlacek, F. Lopour, Future Prospects for Defect and Strain Analysis in the SEM via Electron Channeling, Microsc. Today 20 (2012) 12-16.

[23] J.K. Hite, M.A. Mastro, C.R. Eddy Jr., Approach for dislocation free GaN epitaxy, J. Cryst. Growth 312 (2010) 3143-3146.

[24] G. Naresh-Kumar, B. Hourahine, P.R. Edwards, A.P. Day, A. Winkelmann, A. J. Wilkinson, P.J. Parbrook, G. England, C. Trager-Cowan, Rapid nondestructive analysis of threading dislocations in wurtzite materials using the scanning electron microscope, Phys. Rev. Lett. 108 (2012) 135503.

[25] N. Ferralis, J. Kawasaki, R. Maboudian, C. Carraro, Evolution in surface morphology of epitaxial graphene layers on $\mathrm{SiC}$ induced by controlled structural strain, Appl. Phys. Lett. 93 (2008) 191916. 
[26] I. Gutierrez-Urrutia, S. Zaefferer, D. Raabe, Electron channeling contrast imaging of twins and dislocations in twinning-induced plasticity steels under controlled diffraction conditions in a scanning electron microscope, Scr. Mater. 61 (2009) 737-740

[27] S. Zaefferer, N.-N. Elhami, Theory and application of electron channelling contrast imaging under controlled diffraction conditions, Acta Mater. 75 (2014) 20-50.

[28] F.J. Humphreys, Review Grain and subgrain characterisation by electron backscatter diffraction, J. Mater. Sci. 36 (2001) 3833-3854.

[29] F.J. Humphreys, Y. Huang, I. Brough, C. Harris, Electron backscatter diffraction of grain and subgrain structures-resolution considerations, J. Microsc. 195 (1999) 212-216.

[30] S. Wright, M. Nowell, J. Basinger, Precision of EBSD based orientation measurements, Microsc. Microanal. 17 (2011) 406-407.

[31] S.I. Wright, M. Nowell, High-speed EBSD, Adv. Mater. Process. 166 (2008) 29-31.

[32] Y.N. Picard, M.E. Twigg, J.D. Caldwell, C.R. Eddy, P.G. Neudeck, A.J. Trunek, J. A. Powell, Electron channeling contrast imaging of atomic steps and threading dislocations in 4H-SiC, Appl. Phys. Lett. 90 (2007) 234101.
[33] Y.N. Picard, M.E. Twigg, J.D. Caldwell, C.R. Eddy Jr, M.A. Mastro, R.T. Holm, Resolving the Burgers vector for individual GaN dislocations by electron channeling contrast imaging, Scr. Mater. 61 (2009) 773-776.

[34] R. Kamaladasa, W. Jiang, Y. Picard, Imaging dislocations in single-crystal SrTiO3 substrates by electron channeling, J. Electron. Mater. 40 (2011) 2222-2227.

[35] H. Mansour, J. Guyon, M.A. Crimp, N. Gey, B. Beausir, N. Maloufi, Accurate electron channeling contrast analysis of dislocations in fine grained bulk materials, Scr. Mater.84-85 (2014) 11-14.

[36] M.G. Hall, G.K. Skinner, A spiral scanning attachment for electron-channelling studies with a scanning electron microscope, J. Phys. E: Sci. Instrum. 11 (1978) 1129.

[37] D.C. Joy, D.E. Newbury, Scanning electron microscope selected area channelling patterns from $1 \mu \mathrm{m}$ specimen areas, J. Mater. Sci. 7 (1972) 714-716. 